\title{
MODELO EXPLORATÓRIO PARA A PROSPECÇÃO DE DEPÓSITOS DE Zn-Pb UTILIZANDO DADOS DE ALTA RESOLUÇÃO ESPACIAL E ESPECTRAL DO SENSOR GEOSCAN MKII: ESTUDO DE CASO NO DEPÓSITO SALOBRO, PORTEIRINHA (MG)
}

\author{
TATI DE ALMEIDA ${ }^{1}$, CARLOS ROBERTO DE SOUZA FILHO' ${ }^{1}$, FRANCISCO ROBÉRIO DE \\ ABREU ${ }^{2}$, ALVARO PENTEADO CRÓSTA ${ }^{1}$
}

\begin{abstract}
AN EXPLORATION MODEL FOR DETECTION OF Zn-Pb DEPOSITS USING GEOSCAN MK-II'S HIGH SPATIAL AND SPECTRAL RESOLUTION DATA: A CASE STUDY IN THE SALOBRO DEPOSIT, PORTEIRINHA (MG) The goal of this paper was two-folded. Firstly, based on the available literature, descriptive models were compiled for generic stratabound, sedimentaryhosted $\mathrm{Zn}-\mathrm{Pb}$ deposits, and optimum spectral bandwidths covered by contemporary multispectral sensors were suggested for detection of their primary geologic features. Secondly, the detection model was tested in the Salobro $\mathrm{Zn}-\mathrm{Pb}$ deposit (N-NE portion of the Minas Gerais State), considering GEOSCAN data that consist of 24 spectral bands at $5 \mathrm{~m}$ spectral resolution. Before the conceptual detection model was tested in the Salobro deposit, a geologic surveying followed by petrographic and spectral analysis were accomplished in order to tune the model to a list of local geologic observational phenomena at the surface (e.g., favourable host rocks, alteration patterns, structural controls) that might lend themselves to remote sensing investigation. The list of observational phenomena were then filtered by a set o physical environmental constraints (climate, vegetation and soil cover) to produce a new set of landscape attributes (detectable phenomena) that stood a reasonable chance of being detected and exploited in this particular study area. Dense vegetation (even in dry seasons) and soil (either in situ or transported) cover most of rocks throughout the area of the deposit, which limits considerably the observational features, screening the detectable features to a few. Among the main detectable features are the $\mathrm{Zn}-\mathrm{Pb}$ ore zone (ferruginous metachert) and banded iron formations closely associated to it. Key spectral bandwidths to detecting these two sets of rocks and that are simultaneously available within GEOSCAN data, comprise : (i) 400-950nm-covering the visible and near infrared region of the spectrum, for mapping iron oxides and hydroxides; and (ii) $8500-12500 \mathrm{~nm}-$ covering the thermal region, for mapping silica-rich rocks. A reasoned thematic mapping approach, favoured in this study, tailored image processing of GEOSCAN data to the specific attributes of interest, focusing on the detectable features yielded from the model. In this view, image processing was split in two steps: (i) a basic toolkit for image processing, including colour composite images, band ratios and principal component transformations, were applied to the data aiming to discriminate between the key rocks of the deposit; (ii) spectral classifiers (SAM and SFF) were then employed to identify such rocks based on spectral libraries. As predicted by the detection model, both sets of techniques, particularly the ordinary ones (e.g., band $20-9170 \mathrm{~nm} \pm 530 \mathrm{~nm}$; band $14-2176 \mathrm{~nm} \pm 44 \mathrm{~nm}$ and band $6-740 \mathrm{~nm} \pm 23 \mathrm{~nm}$, in RGB, merged with the digital elevation model of the area), were able to successfully map the surface expression of the ore zone and the banded iron formations within the deposit. However, most of the other geologic features associated to the deposit was Masked by vegetation and soil cover. This paper has demonstrated that theoretical exploration models based on remote sensing data can sufficiently support the indirect targeting of base metal deposits. However, the physical environment at the surface, as well as the choice of remote sensing data, may constrain the suitability of the model for a particular scale. Using the Salobro deposit as a control, this work showed that the application of a specific detection model, coupled with the moderately high spatial and spectral resolution of GEOSCAN data, was able to frame the ore zone accurately, an achievement that has been rarely repeated in tropical terrains.
\end{abstract}

Keywords: remote sensing, GEOSCAN imagery, mineral exploration, $\mathrm{Zn}-\mathrm{Pb}$ deposits.

Resumo A utilização de um modelo prospectivo voltado às características geológicas e espectrais das unidades litológicas do depósito Salobro permitiu a detecção, por sensoriamento remoto multiespectral, de um horizonte mineralizado em $\mathrm{Zn}$ - $\mathrm{Pb}$, previamente reconhecido nos trabalhos de campo desenvolvidos pela Docegeo-CVRD. O depósito Salobro, localizado na porção N/NE do Estado de Minas Gerais, é composto por rochas metassedimentares pertencentes à Seqüência Salobro. Esta seqüência é subdividida em 3 unidades: Unidade A (quartzo-muscovita xistos grossos); Unidade B (metaconglomerados, anfibólio xistos laminados, horizonte mineralizado e formações ferríferas bandadas) e Unidade C (muscovita-clorita-quartzo xistos). As rochas associadas e hospedeiras, $\mathrm{o}$ controle estratigráfico, o tipo de minério e as estruturas reconhecidas no depósito Salobro, indicam que a mineralização de $\mathrm{Zn}-\mathrm{Pb}$ é similar àquelas classificadas como do tipo estratiforme hospedadas em sedimentos (EHS), metamorfisada em grau anfibolito médio e retro-metamorfisada no fácies xisto verde alto. As características geológicas da região do depósito e a análise petrográfica e espectral das unidades aflorantes permitiram a confecção de um modelo exploratório baseado em dados e técnicas de sensoriamento remoto, onde foram enfocados, principalmente: (i) rochas encaixantes e minerais de minério, (ii) alteração primária e secundária no pipe, (iii) controle do minério, e (iv) estrutura associada. O modelo exploratório foi aplicado a imagens de alta resolução espacial (5m) e espectral

1 - Universidade Estadual de Campinas, Instituto de Geociências, Caixa Postal 6152, 13083-970, Campinas, São Paulo, E-mail: tati_almeida@pop.com.br; beto@ige.unicamp.br, alvaro@ige.unicamp.br

2 - 317 Lexington Ave. \#258, San Antonio, Texas, USA, 78215. E-mail: frandeabreu@earthlink.net 


\begin{abstract}
(24 bandas, abrangendo o visível, infravermelho próximo, infravermelho de ondas curtas e infra-vermelho termal) obtidas pelo sensor GEOSCAN MKII em agosto de 1992. Estas imagens foram processadas por dois conjuntos de técnicas: um primeiro voltado ao realce e discriminação entre materiais geológicos (composições coloridas, operações aritméticas e principais componentes); e um segundo, visando a identificação sistemática (classificação espectral) desses materiais ( $S A M$ e $S F F)$. O processamento das imagens GEOSCAN do depósito Salobro através dessas técnicas possibilitou a delimitação da expressão superficial das formações ferríferas bandadas e do horizonte metachert ferruginoso (horizonte mineralizado em $\mathrm{Zn}-\mathrm{Pb}$ ). A utilização do modelo digital do terreno (MDT) permitiu a fusão destas imagens à topografia da área e demonstrou que a melhor resposta espectral desses litotipos encontra-se nos altos topográficos, onde há pouco ou nenhum solo transportado e pouca influência da vegetação. Como base nos resultados obtidos nessa pesquisa, foi demonstrado que modelos exploratórios baseados em dados e técnicas de sensoriamento remoto podem ser importantes na prospecção de depósitos de metais base, desde que os aspectos fisiográficos do alvo sejam bem conhecidos e incorporados na estratégia de prospecção.
\end{abstract}

Palavras-chave: sensoriamento remoto, imagens GEOSCAN, exploração mineral, depósitos de Zn-Pb.

INTRODUÇ̃̃O Cerca de $75 \%$ das reservas mundiais de $\mathrm{Zn}-\mathrm{Pb}$ são encontradas em depósitos do tipo "estratiforme, hospedado em sedimentos" (Holm et al. 1999). Apesar de bem descritos na literatura, pouco foi realizado no sentido de demonstrar quais, dentre as suas características, podem ser convenientemente detectadas por sensoriamento remoto multiespectral (Spatz \& Wilson 1997). Isso resulta na falta de uma estratégia para a prospecção de $\mathrm{Zn}-\mathrm{Pb}$, utilizando sensoriamento remoto, também sentida na busca de outros tipos de depósitos, como enfocado por diversos autores (e.g., Largie et al. 1993, Spatz 1997, Spatz \& Wilson 1997, Spatz 1999, Swalf 2000, Almeida 2000).

No Brasil, a descoberta de depósitos estratiformes de $\mathrm{Zn}-\mathrm{Pb}$ hospedados em sedimentos é limitada a poucos casos, dos quais destacam-se: (i) as antigas minas do Perau e Canoas, no distrito mineiro do Vale da Ribeira, Paraná (Daitx 1996); (ii) a mina desativada de Boquira, na região central da Bahia (Fleischer e Espourteille, 1999); (iii) a mina de Vazante, na região oeste de Minas Gerais (Dardenne e Freitas-Silva, 1999); e o deposito Salobro, recentemente descoberto pela DOCEGEO na região norte de Minas Gerais (Abreu \& Oliveira 1998). O depósito Salobro possui, segundo DOCEGEO (2001), reservas medidas e inferidas da ordem de 6,5 milhões de toneladas de minério, com teor médio de $\sim 7 \%$ de $\mathrm{Zn}+\mathrm{Pb}$.

No presente estudo, o depósito Salobro foi utilizado como área teste para a confecção de um modelo prospectivo de depósito Zn$\mathrm{Pb}$ do tipo "estratiforme, hospedado em sedimentos"(EHS), usando dados de sensoriamento remoto em áreas tropicais. A escolha deste depósito foi baseada nos seguintes critérios: (i) potencial econômico; (ii) características fisiográficas, típicas de terrenos tropicais (i.e. cobertura vegetal e perfil intempérico), o que pode tornar o modelo prospectivo de aplicação mais ampla; (iii) existência de mapeamento geológico detalhado; (iv) existência de dados multiespectrais de alta resolução espacial (5m) e espectral (24 bandas), coletados pelo sensor GEOSCAN.

\section{CARACTERÍSTICASDETECTÁVEISPORSENSORIAMENTO REMOTO EM DEPÓSITOS DE Zn-Pb DO TIPO EHS Os} depósitos do tipo EHS, metamorfisados ou não, ocorrem intercalados a seqüências sedimentares clásticas, químicas e/ou biogênicas, onde a contribuição de rochas vulcânicas é muito pequena ou nula. Este tipo de depósito é associado a rifts intracontinentais ou a falhas de grábens em margens continentais (Goodfellow et al. 1993, MacIntyre 1995) e, segundo Gustafson \& Willians (1981), ocorre em maior freqüência em terrenos de idades Proterozóica e Fanerozóica.

Os corpos de sulfeto dos depósitos EHS ocorrem como camadas e lentes e apresentam estruturas maciça, bandada ou disseminada (Guilbert \& Park 1981, Gustafson \& Williams 1981, Goodfellow et al. 1993). As camadas de sulfetos têm espessura centimétrica à métrica e extensão quilométrica. A razão entre a extensão lateral e espessura dos corpos é freqüentemente superior a 20 (Largie 1983). O principal sulfeto presente é a pirita, porém em alguns casos predomina a pirrotita. Os minerais de minério são a esfalerita, galena e a calcopirita (Goodfellow et al. 1993), que distribuem-se, do centro para as bordas, zonados de acordo com a seguite ordem: $\mathrm{Cu} \rightarrow \mathrm{Pb} \rightarrow \mathrm{Zn} \rightarrow \mathrm{Fe}$. Porém, em depósitos onde a quantidade de $\mathrm{Fe}$ presente é pequena, a zonação observada é $\mathrm{Pb} \rightarrow \mathrm{Zn} \rightarrow \mathrm{Ba}$ (Gustafson \& Williams 1981). Os corpos de minério distribuem-se em quatro dominios principais, compartimentados em relação ao vent hidrotermal, como: (i) fácies sedimentar hidrotermal (ou fácies de minério acamadado); (ii) fácies sedimentar distal; (iii) vent complex e; (iv) feeder pipe (Goodfellow et al. 1993). No entanto, dependendo das relações topográficas, relações de transporteconcentração e diluição-dispersão, as concentrações de sulfetos maciços podem ser depositadas a dezenas de metros do canal abastecedor do sistema hidrotermal (Guilbert \& Park 1986, Wilton 1998).

Utilizando as características supracitadas, os dados e resultados apresentados nos trabalhos de Chenkui et al. (1991), Largie et al. (1993), Spatz (1996, 1997, 1999), Spatz \& Wilson (1997), Swalf(2000) e Almeida (2000), e as bibliotecas espectrais digitais da USGS (http://speclab.cr.usgs.gov), foram listadas, na Tabela 1, as características destes depósitos passíveis de detecção por sensoriamento remoto e os intervalos espectrais correlacionáveis a cada uma delas.

Vale salientar que este modelo é hipotético e que a influência de fatores externos, tais como vegetação, clima, topografia, iluminação, espalhamento atmosférico, entre outros, não foi considerada. Desta forma, o modelo, antes de aplicado, deve ser necessariamente adaptado, levando-se em consideração estes fatores externos, os quais sempre serão determinantes para o sucesso ou fracasso de aplicações de sensoriamento remoto em exploração mineral.

Em áreas tropicais, por exemplo, onde as superfícies são dominadas por densa cobertura vegetal e espesso manto de intemperismo, as respostas espectrais dos materiais primários, diagnósticos de depósitos de Zn-Pb EHS, podem ser totalmente mascaradas. De modo a contornar essas limitações, a época de aquisição de imagens precisa ser considerada. Na estação seca, aumenta a possibilidade de aquisição de imagens do substrato exposto, função da perda substancial de massa foliar por árvores decíduas. Além disto, ambigüidades espectrais causadas por níveis de solos transportados ou horizontes coluvionares e depositados sobre materiais "in situ" podem ser minimizadas, concentrando a triagem de alvos nos altos topográficos. As assinaturas espectrais destes altos devem corresponder à resposta do 
Tabela 1 - Principais características dos depósitos de Zn-Pb estratiformes hospedados em rochas sedimentares e intervalos espectrais e resoluções espaciais necessárias para a sua detecção por sensoriamento remoto.

\begin{tabular}{|c|c|c|c|c|c|}
\hline \multirow{2}{*}{\multicolumn{2}{|c|}{$\begin{array}{c}\text { Depósitos de Zn-Pb Estratiformes Hospedados } \\
\text { em Rochas Sedimentares }\end{array}$}} & \multicolumn{2}{|c|}{$\begin{array}{l}\text { Posições espectrais } \\
(\mu \mathrm{m})\end{array}$} & \multicolumn{2}{|c|}{$\begin{array}{l}\text { Res. Espacial } \\
(\mathrm{m})\end{array}$} \\
\hline & & Multiespec & Hiperespec & Recon. & Dep. $^{2}$ \\
\hline Rochas associadas & $\begin{array}{l}\text { Rochas sedimentares argilíticas, calcárias, e, raramente, } \\
\text { quartzíticas. }\end{array}$ & $\begin{array}{l}\text { VNIR, } \\
\text { SWIR } \\
9.5 / 10.5\end{array}$ & $\begin{array}{c}2.33 \\
9.7\end{array}$ & $20-30$ & $10-20$ \\
\hline $\begin{array}{l}\text { Minerais de Minério } \\
\text { (princ. e subord.) }\end{array}$ & $\begin{array}{l}\text { Py, Po, } \mathrm{Sp}, \mathrm{Ga}, C c \text { e } \text { Brt. Traços de marcassita, } \\
\text { arsenopirita, bismutinita, molibidenita, enargita, } \\
\text { milerita, fribergita, cobaltita, vallerita, melnikovita. }\end{array}$ & $0.7-0.9$ & 0.85 & $10-20$ & $5-8$ \\
\hline \multicolumn{6}{|c|}{ Depende da rocha hospedeira do minério } \\
\hline $\begin{array}{l}\text { Minerais de } \\
\text { alteração }\end{array}$ & $\begin{array}{c}\text { Sílica, turmalina, carbonato, albita e clorita de } \\
\text { ambientes de baixa temperatura. Barita e argilas com } \\
\text { amônia são citados em alguns depósitos }\end{array}$ & $2.3-2.4$ & $\begin{array}{l}2.24 ; 2.29 \\
\quad 2.32\end{array}$ & $10-15$ & $5-7$ \\
\hline $\begin{array}{l}\text { Estruturas } \\
\text { associadas }\end{array}$ & $\begin{array}{c}\text { Falhas regionais normais de alto ângulo, anticlinais com } \\
\text { falhas locais e normais associadas }\end{array}$ & \multicolumn{2}{|c|}{ VNIR; SWIR } & $20-80$ & $10-20$ \\
\hline Controle do minério & $\begin{array}{c}\text { Seqüências sedimentares favoráveis, estruturas rúpteis } \\
\text { de grande porte }\end{array}$ & $\begin{array}{l}\text { VNIR } \\
\text { SWIR }\end{array}$ & $\begin{array}{l}\text { VNIR } \\
\text { SWIR }\end{array}$ & $30-40$ & $15-20$ \\
\hline $\begin{array}{l}\text { Alteração primária } \\
\text { no pipe (vertical) }\end{array}$ & $\begin{array}{c}\text { Estreita zona com jasperóide, sílica, e pouca illita, } \\
\text { caulinita, montmorillonita, com descalcificação. } \\
\text { Ocorrência distal de amônia. }\end{array}$ & $\begin{array}{l}2.15-2.35 \\
9.5-10.5\end{array}$ & $\begin{array}{l}2.19,2.21 \\
2.33,2.34 \\
\quad 9.7\end{array}$ & $15-30$ & $5-8$ \\
\hline Alteração secundária & Goethita e pouca jarosita. & $\begin{array}{l}0.6-0.7 \\
0.75-1.0\end{array}$ & 9,$0 ; 9,5$ & $10-30$ & $5-8$ \\
\hline Zonação lateral & $\begin{array}{c}\text { Silicificação com illita em gradação para abundância } \\
\text { em illita-caulinita }\end{array}$ & $\begin{array}{l}9.5-10.5 \\
2.15-1.35\end{array}$ & $\begin{array}{c}2.19 ; 2.21 \\
9.7\end{array}$ & $10-20$ & $4-6$ \\
\hline
\end{tabular}

substrato, mesmo que este se encontre parcial ou totalmente alterado por intemperismo.

LOCALIZAÇÃOEGEOLOGIADODEPÓSITOSALOBRO A área de estudo localiza-se a $15 \mathrm{Km}$ à oeste da cidade de Porteirinha, situada no extremo norte do Estado de Minas Gerais (Fig. 1a) e encontra-se inscrita na Folha de Janaúba (SD-23-Z-D-IV, escala $1: 100.000)$.

$\mathrm{O}$ arcabouço geológico da borda leste do Cráton do São Francisco entre Itacambira e Monte Azul, centro-norte de Minas Gerais, é controlado por uma janela estrutural (Bloco ItacambiraMonte Azul - BIMA) que possibilita a exposição de rochas magmáticas e metamórficas, formadas até o final da orogênese Tranzamazônica (Drumond et al. 1980, Crocco-Rodrigues et al. 1992). Guimaraes et al. (1993), subdividem as rochas deste bloco, entre a vila de Barrocão e a cidade de Porteirinha, em 6 unidades: Complexo Metamórfico Córrego do Cedro, que engloba gnaisses bandados de composição granitica; Grupo Riacho dos Machados, constituído por xistos, anfibolitos e raras formações ferriferas; Suíte Granitóide Gurutuba, de composição tonalitica; Suíte Granitóide Pedra do Urubu; Suíte Monzonítica Paciência e Suíte Granítica Confisco (Fig. 1b).

Santos \& Paez (1992) mapearam, em escala de semi-detalhe, uma faixa do Grupo Riacho dos Machados que ocorre isolada entre as cidades de Porteirinha e Janaúba, denominando-a informalmente de Seqüência Salobro (Fig. 1b). Segundo Abreu \& Oliveira (1998), trabalhos de prospecção geoquímica possibilitaram a identificação de anomalias de $\mathrm{Zn}$ e $\mathrm{Pb}$ associadas a terrenos incluídos nessa seqüência. Com o detalhamento destas anomalias foi definido o depósito Salobro, no qual ocorre um horizonte de metachert ferruginoso com porções ricas em $\mathrm{Zn}$ e valores anômalos de $\mathrm{Pb}$ (Abreu \& Oliveira 1998). Estes mesmos trabalhos em escala de detalhe possibilitaram a subdivisão da seqüência Salobro nas unidades informais A, B e C (Fig. 2), descritas a seguir.

Unidade A Unidade basal constituída predominantemente por quartzo-muscovita xistos. As paragêneses minerais, as relações intergrãos e o bandamento presentes nestes xistos sugerem que os mesmos correspondem a sedimentos alúmino-silicáticos que foram submetidos a metamorfismo em grau anfibolito, com posterior retrometamorfismo na fácies xisto verde.

Unidade B Constituída por três tipos litológicos principais: anfibólio-xistos laminados, metachert feruginoso e formações ferríferas bandadas. Os anfibólio-xistos laminados apresentam variações composicionais de acordo com a porção estratigráfica em que se encontram, de modo que não existe um único litotipo, mas sim uma intercalação heterogênea entre lâminas e bandas milimétricas de vários tipos de "xistos" e/ou "fels", com predominância constante de anfibólios (tremolita/ actinolita) e minerais do grupo do epidoto. $\mathrm{O}$ topo do pacote é marcado por um nível de metachert ferruginoso que corresponde a uma rocha quartzosa, com grande quantidade de estruturas box-work em superfície, às quais se associam valores anômalos de $\mathrm{Pb}$ e $\mathrm{Zn}$ em amostras de rocha e solo. Esse último litotipo sustenta relevo de crista alongado na direção WSW-ESSE. No conjunto pode-se interpretar as composições, texturas e estruturas presentes no anfibólio xisto laminado como resultantes do metamorfismo de sedimentos químico-detríticos, submetidos à fácies anfibolito e retrometamorfisado na fácies xisto verde.

As formações ferríferas bandadas são constituídas predominantemente por intercalações submilimétricas entre niveis quartzosos e niveis de magnetita. Essas rochas também sustentam cristas alongadas de direção WSW-ENE e se intercalam vertical e lateralmente com os xistos da Unidade C.

Os tipos litológicos da Unidade B caracterizam um ambiente deposicional com forte contribuição hidrotermal, com a qual a mineralização de $\mathrm{Zn}$ pode estar geneticamente associada.

Unidade C No topo da seqüência, essa unidade é composta de muscovita-clorita-quartzo xistos bandados, localmente com pseudomorfos de cristais de estaurolita substituídos por sericita. $\mathrm{O}$ bandamento presente geralmente mostra granodecrescência, $\mathrm{o}$ 
Modelo exploratório para a prospecção de depósitos de Zn-Pb utilizando dados de alta resolução espacial e espectral do sensor Geoscan MKII: estudo de cado no depósito Salobro, Porteirinha (MG)
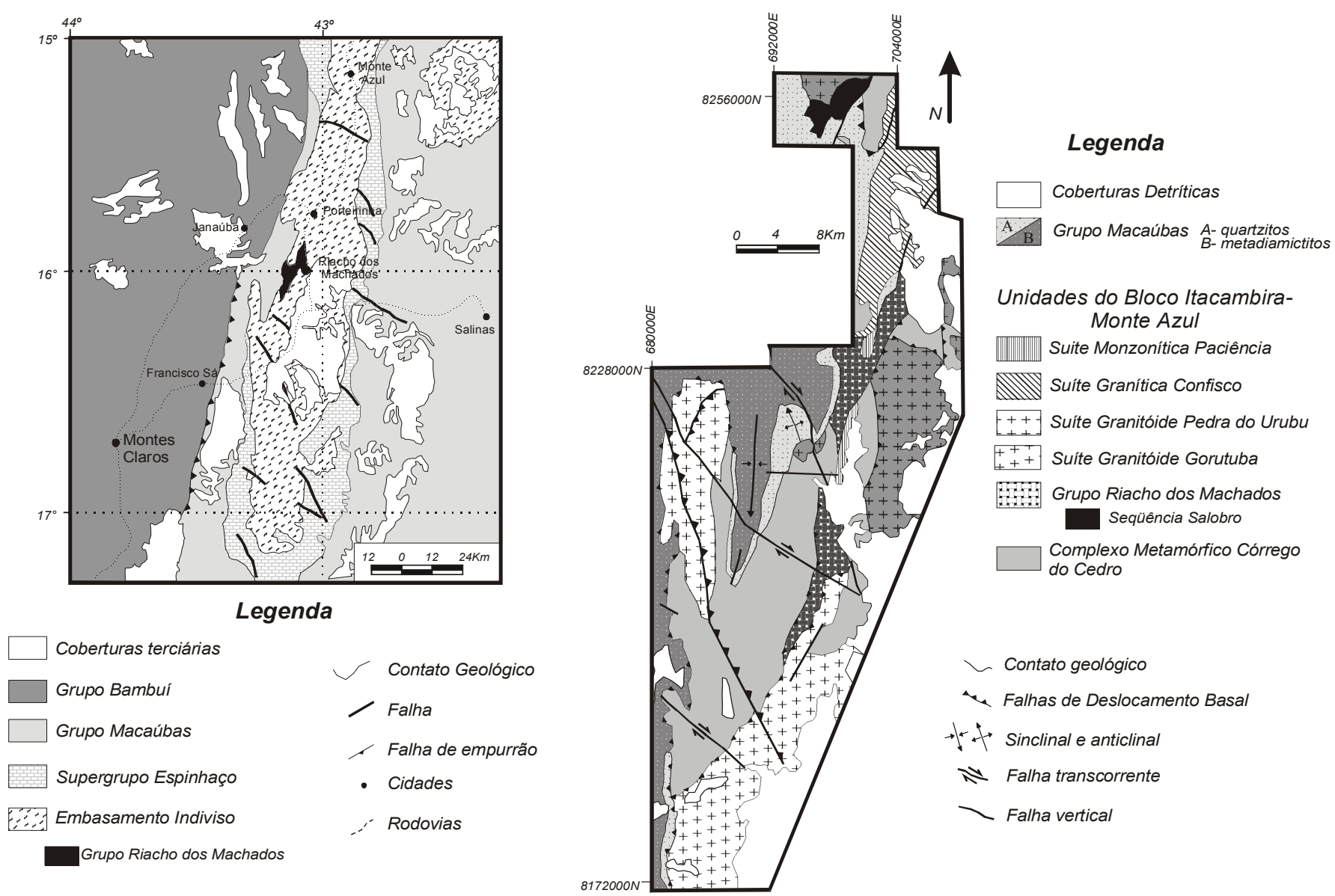

Figura 1 - (a) Mapa de localização e geologia regional do Bloco Itacambira- Monte Azul (BIMA) no contexto da Faixa de Dobramento Araçuai (Guimarães et al. 1993). (b) Mapa geológico do BIMA entre os municípios de Itacambira e Monte Azul (Guimarães et al. 1993).

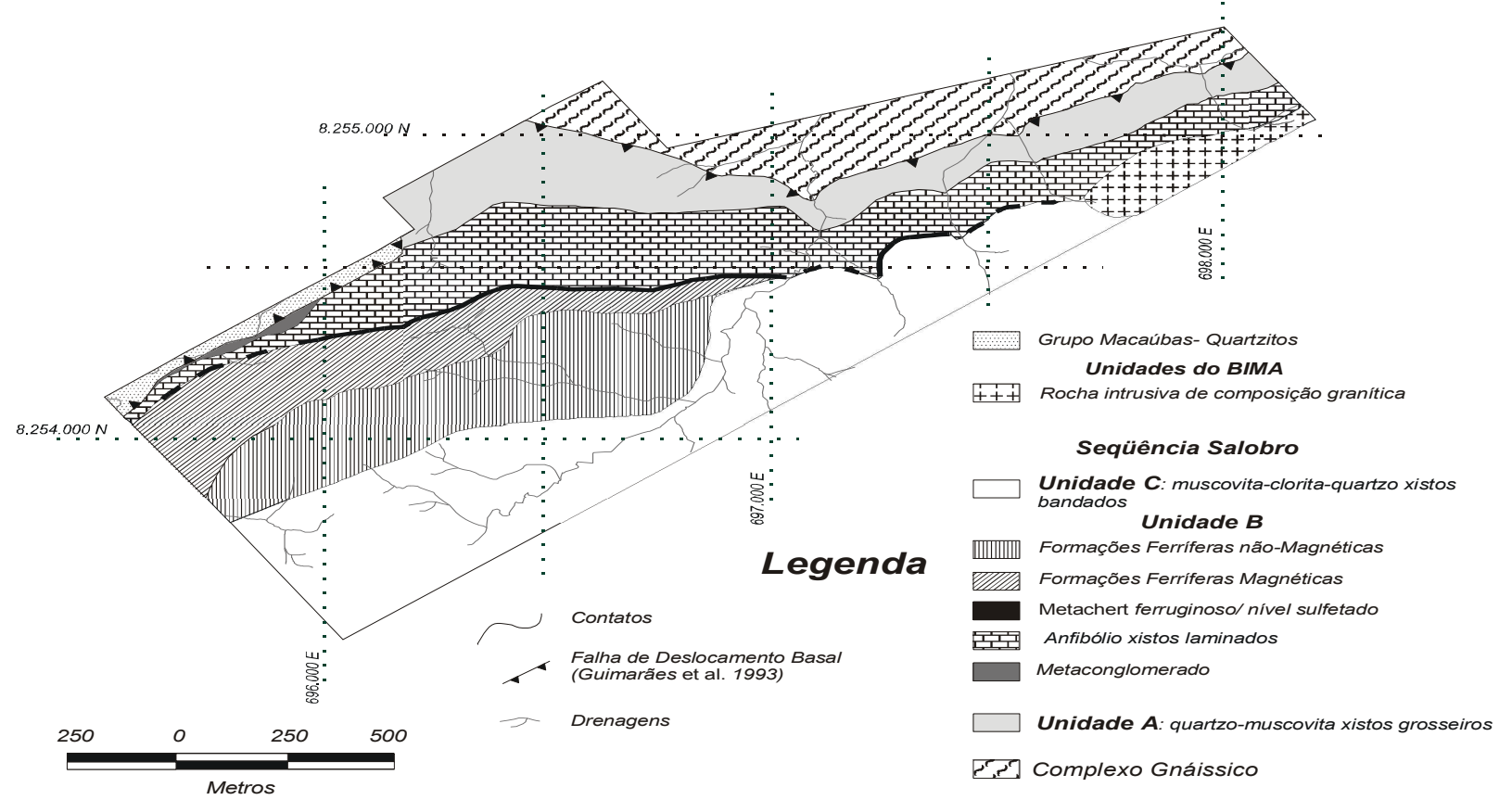

Figura 2 - Mapa geológico do depósito Salobro (modificado DOCEGEO 1999). 
que, junto às parageneses minerais, permite a inferência de um protolito turbidítico, posteriormente metamorfisado na fácies anfibolito e retrometamorfisado na fácies xisto verde.

MATERIAIS E MÉTODOS Imagens do sensor aeroportado GEOSCAN MKII As imagens utilizadas neste trabalho foram adquiridas no mês de setembro do ano de 1992, utilizando o sensor multiespectral aerotransportado GEOSCAN MK-II. Esse imageador possui 46 bandas espectrais dentre as quais 24 podem ser selecionadas simultaneamente para a captação dos dados entre 450 e $12.000 \mathrm{~nm}$, abrangendo as regiões do visível (VIS), infravermelho próximo (NIR), infravermelho de ondas curtas (SWIR) e infravermelho termal (TIR) (Tabela 2). O campo de visada desse sensor é de $\pm 45^{\circ}$ com um campo de visada instantânea de 2.1 a 3.0 miliradianos. Os intervalos espectrais específicos dos dados coletados neste levantamento encontram-se listados na Tabela 2.

Uma das características deste sensor é que o intervalo dinâmico de cada banda espectral é estabelecido através de ajustes de ganho e offset (PROSPEC 1993). Para determinar este ajuste é realizado um vôo preliminar sobre a área, estabelecendo-se a intensidade máxima e mínima para cada banda e redistribuindo-se os valores no intervalo dinâmico de 8 bits. Este procedimento, embora facilite o ajuste ótimo do histograma das imagens, inibe a utilização de técnicas simples de correção atmosférica. Visto que esta correção é crítica para o sucesso da aplicação de técnicas de processamento mais sofisticadas (i.e., classificação espectral), utilizou-se neste estudo um algorítmo desenvolvido para amenizar o efeito do espalhamento atmosférico residual. Este algorítmo é denominado de IARR (Internal Average Relative Reflectance) e já demonstrou sua eficiência para a correção atmosférica de dados GEOSCAN (Du 1996, Fraser \& Agar 1997, Agar \& Pávez 1999). A técnica IARR é utilizada principalmente para normalizar imagens de áreas onde o comportamento espectral dos alvos como um todo é pouco conhecido (Roberts et al. 1985, Conel et al. 1985, Kruse 1990). O algoritmo gera um espectro médio considerando todos os pixels da imagem sob estudo, sendo em seguida dividido pelo espectro de cada pixel dessa imagem.

Espectrorradiômetro FieldSpec Full Resolution (FR) Com o advento de espectrômetros portáteis de alta resolução espectral, cada vez mais a espectroscopia de reflectância está sendo difundida tanto para auxiliar o complexo entendimento entre as interações matéria e energia, base para o uso do sensoriamento remoto (Maracci 1992, Curtiss \& Goetz 1994), como para o auxílio à identificação de minerais, mapeamento de zonas de alteração hidrotermal, estudos cristalográficos, entre outros (Duke 1994, Martines-Alonso et al. 1997 in Passos 1999).

O instrumento utilizado nesse estudo, o espectroradiômetro FieldSpec Full Resolution, detecta radiação eletromagnética no intervalo espectral entre $350 \mathrm{~nm}$ e $2500 \mathrm{~nm}$. Esse instrumento foi utilizado para as medições dos materiais geológicos presentes no depósito Salobro, sob fonte artificial de iluminação (lâmpada halógena de $3000^{\circ} \mathrm{K}$ de temperatura) e lente redutora do campo de visada de $1^{\circ}$, posicionada a uma distância de aproximadamente $20 \mathrm{~cm}$ do material alvo, o que proporcionou um campo de visada instantânea de $0,35 \mathrm{~cm}$. A calibração do aparelho foi realizada através de uma placa de referência, constituída por um composto ótico sintético (Spectralon), que se comporta como uma superfície lambertiana. A coleta e o processamento dos dados foram efetuados com o auxílio de um lap top e software controlador. Este software, opcionalmente, trabalha integrado ao Spectral Angle Mapper (SAM, Kruse et al. 1993), um programa capaz de analisar automaticamente o conteúdo mineralógico de uma curva espectral, com base em bibliotecas espectrais de referência, tais como a do United States Geological Survey - USGS (http://speclab.cr.usgs. gov/spectral-lib.html).

A figura 3 resume as principais etapas desenvolvidas durante a realização da pesquisa, que envolveu duas etapas principais: (i) levantamento bibliográfico das características, detectáveis por sensoriamento remoto, de depósitos tipo de $\mathrm{Zn}-\mathrm{Pb}$ estratiforme hospedado em sedimentos e; (ii) adaptação deste modelo teórico em um caso prático no depósito Salobro.

\section{ASSINATURAS ESPECTRAIS DOS LITOTIPOS DO} DEPÓSITO SALOBRO A espectroscopia de reflectância é um método que não requer preparação de amostras, e que reúne rapidez e economicidade na caracterização de minerais e rochas. No entanto, como já discutido nos trabalhos de Passos (1999) e Almeida (2000), é necessário um rigoroso controle da mineralogia,

Tabela 2 - Especificações das bandas do sensor GEOSCAN MKII para o aerolevantamento de Riacho dos Machados (fonte: PROSPEC 1993 ).

\begin{tabular}{|c|c|c|c|c|c|c|c|}
\hline \multicolumn{2}{|c|}{ Banda } & \multirow{2}{*}{$\begin{array}{c}\text { Comprimento de onda } \\
\text { central (nm) }\end{array}$} & \multirow{2}{*}{$\begin{array}{c}\begin{array}{c}\text { Largura da banda } \\
\text { (nm) }\end{array} \\
42\end{array}$} & \multicolumn{2}{|c|}{ Banda } & \multirow{2}{*}{$\begin{array}{c}\begin{array}{c}\text { Comprimento de } \\
\text { onda central (nm) }\end{array} \\
2136\end{array}$} & \multirow{2}{*}{$\begin{array}{c}\begin{array}{r}\text { Largura da } \\
\text { banda (nm) }\end{array} \\
44\end{array}$} \\
\hline 1 & \multirow{10}{*}{ 兰 } & & & 13 & \multirow{6}{*}{$\underset{n}{\mathscr{B}}$} & & \\
\hline 2 & & 583 & 67 & 14 & & 2176 & 44 \\
\hline 3 & & 645 & 71 & 15 & & 2220 & 44 \\
\hline 4 & & 693 & 24 & 16 & & 2264 & 44 \\
\hline 5 & & 717 & 24 & 17 & & 2308 & 44 \\
\hline 6 & & 740 & 23 & 18 & & 2352 & 44 \\
\hline 7 & & 830 & 22 & 19 & \multirow{6}{*}{$\cong$} & 8640 & 530 \\
\hline 8 & & 873 & 22 & 20 & & 9170 & 530 \\
\hline 9 & & 915 & 21 & 21 & & 9700 & 530 \\
\hline 10 & & 955 & 20 & 22 & & 10220 & 533 \\
\hline 11 & & 2044 & 44 & 23 & & 10750 & 533 \\
\hline 12 & & 2088 & 44 & 24 & & 11280 & 533 \\
\hline
\end{tabular}




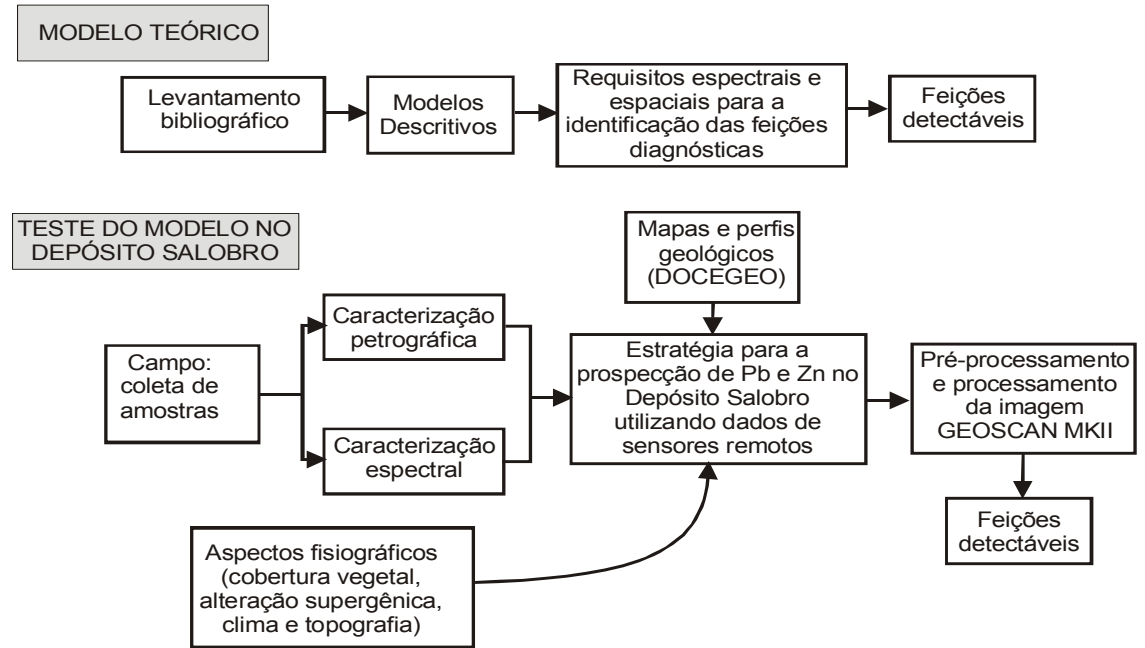

Figura 3 - Fluxograma dos métodos utilizados neste estudo.

por meio de lâminas delgadas, difratometria de Raios-X e/ou microscopia eletrônica em amostras de referência, para uma maior confiabilidade nos resultados obtidos a partir desta técnica.

As curvas de reflectância espectral obtidas com o FieldSpec Full Resollution (FR) foram interpretadas tendo como base os trabalhos de Grove et al. (1992) e Clark (1999), e complementadas com análise automática, proporcionada pelo programa SIMIS Field 2.9 (Spectrometer Independent Mineral Identification Software) (Macklin 1998).

A análise espectral permitiu uma caracterização mineralógica do depósito Salobro que se aproximou bastante daquela obtida a partir da petrografia e de observações feitas no campo. Somente uma pequena parcela (10-20\%) dos dados coletados com o FieldSpec e analisados com o programa SIMIS mostraram pouca ou nenhuma correspondência com os dados petrográficos, admitidos como mais precisos. Os resultados menos satisfatórios do estudo espectral foram obtidos nos anfibólios xistos laminados da Unidade B, não tendo sido possível caracterizar uma assinatura espectral única para este conjunto de xistos devido à sua heterogeneidade composicional.

O intemperismo modificou profundamente a mineralogia original das rochas do depósito Salobro, resultando em misturas de minerais primários com seus correspondentes intempéricos. Contudo, as análises espectrais das unidades desse depósito permitem individualizar os litotipos reconhecidos em três grupos espectrais principais: Grupo I - com feições de absorção entre 400-950 nm; Grupo II - com feições em torno de 2200 nm; e Grupo III - com feições entre $2300 \mathrm{~nm}$ e $2400 \mathrm{~nm}$.

O Grupo I (Fig. 4) compreende as formações ferríferas bandadas (magnéticas e não magnéticas) e o horizonte de metachert ferruginoso. As bandas de absorção desse grupo ocorrem em torno de $400 \mathrm{~nm}$ e, subordinadamente, em $950 \mathrm{~nm}$, relacionadas à presença de hidróxidos e óxidos de ferro (goethita, limonita e hematita). A caracterização espectral de várias amostras intemperizadas do minério do depósito Salobro mostrou que a goethita é o mineral de alteração superficial dominante. De acordo com Fraser et al. (1985) e Raines et al. (1985), a concentração anômala de goethita em gossans (detectado em nossos dados espectrais) pode servir como indicador da presença de jazidas sulfetadas em subsuperfície.

O Grupo II (Fig. 5) engloba rochas ricas em filosilicatos das Unidades A e C. As feições de absorção típicas desse grupo concentram-se em torno de $2200 \mathrm{~nm}$ e decorrem da presença abundante de minerais com íons hidroxila coordenados por íons de alumínio (Al-OH) em sua estrutura, destacando-se os aluminosilicatos do grupo das micas (muscovita e a flogopita) e os argilominerais (caolinita, esmectita e haloisita).

O Grupo III (Fig. 6) compreende rochas ricas em minerais máficos e inclui os litotipos da Unidade B. As feições de absorção características desse grupo concentram-se entre $2300 \mathrm{~nm}$ e $2400 \mathrm{~nm}$ e decorrem de minerais que contem íons hidroxila coordenados por íons de $\mathrm{Mg}(\mathrm{Mg}-\mathrm{OH})$ em sua estrutura, entre os quais estão tremolita/ actinolita, hornblenda e minerais do grupo da clorita.

Os dados indicam que esses três grupos espectrais podem ser detectados por sensores multiespectrais contendo bandas com cobertura independente nessas regiões do espectro (Almeida, 2000) e, portanto, a assinatura espectral de cada um deles pode ser utilizada como guia de prospecção em investigações baseadas em sensoriamento remoto.

\section{MODELO DE DETECÇÃO DO DEPÓSITO SALOBRO} UTILIZANDO OSENSOR GEOSCAN O modelo descritivo e a caracterização do comportamento espectral das feições diagnósticas do depósito Salobro foram sintetizados em um modelo exploratório resumido na Tabela 3. Este "modelo exploratório Salobro" tem como base o sensoriamento remoto multiespectral, com ênfase no sensor GEOSCAN. A Tabela 3 apresenta uma adaptação do modelo exploratório desenvolvido para depósitos de $\mathrm{Zn}-\mathrm{Pb}$ do tipo EHS, de forma genérica, onde foram integradas: (i) as rochas representativas do depósito Salobro; (ii) suas dimensões aflorantes; (iii) os intervalos espectrais onde essas rochas apresentam bandas de absorção diagnósticas; (iv) e quais, dentre as 24 bandas do sensor GEOSCAN, são adequadas à detecção destas variáveis.

Uma característica particular do sensor GEOSCAN é a de possuir bandas espectrais cobrindo a faixa do infravermelho termal (TIR), 


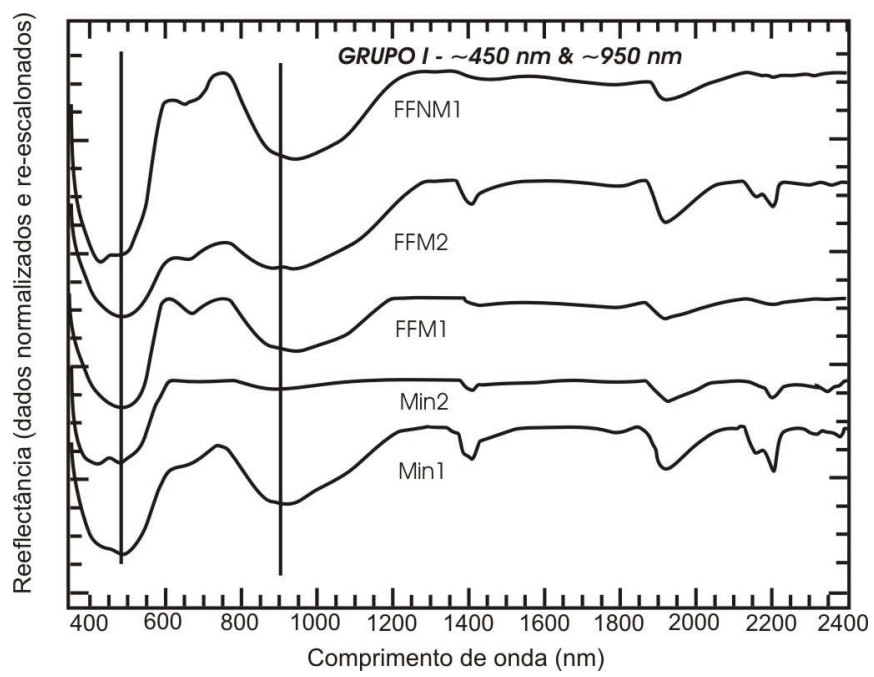

Figura 4 - Grupo Espectral I, com feições de absorção entre 400950nm (Min = amostras de minério de $\mathrm{Zn}-\mathrm{Pb} ; \mathrm{FFM}=$ amostras de formação ferrifera magnética; FFNM = amostra de formação ferrífera não-magnética).

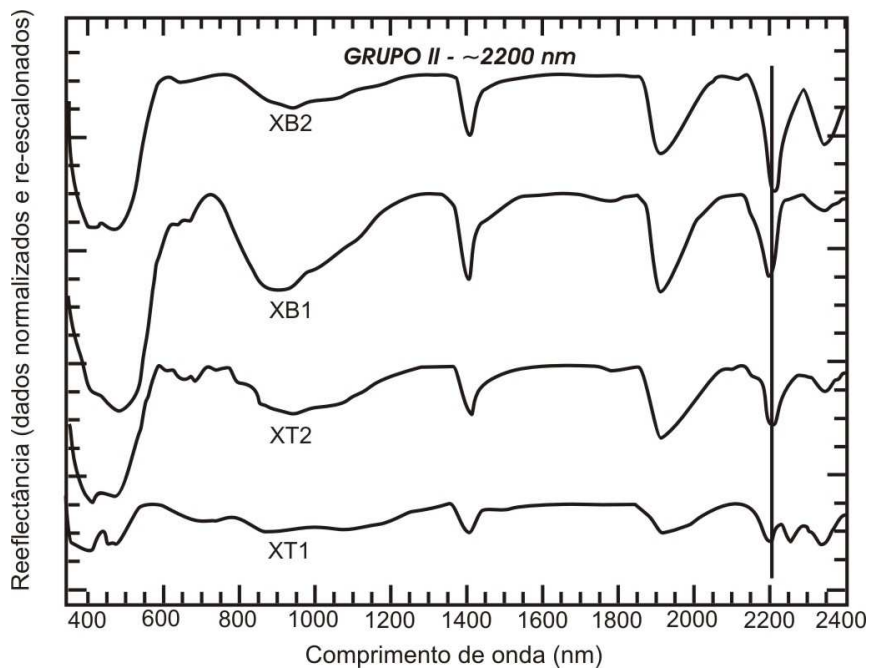

Figura 5 - Grupo Espectral II, com feições de absorção em torno de 2200nm (XB = amostras de quartzo-muscovita xistos da Unidade A; XT = amostras de muscovita-clorita-quartzo xistos da Unidade $C$ )

região onde é possível a detecção de sílica em superfície (Spatz, 1997). Muito embora o espectrorradiômetro FieldSpec FR não seja capaz de medir radiação neste intervalo de comprimentos de onda, o que impediu uma avaliação do comportamento espectral dos materiais geológicos presentes em Salobro nesta faixa do espectro, decidiu-se por incluir o uso destas bandas no modelo exploratório. Esta inclusão foi definida em função da rocha hospedeira do minério (metachert ferruginoso) tratar-se de uma rocha rica em sílica, e, por conseguinte, potencialmente detectável na faixa TIR (Tabela 3 ).

\section{MAPEAMENTO REMOTO DA MINERALIZAÇÃO NO}

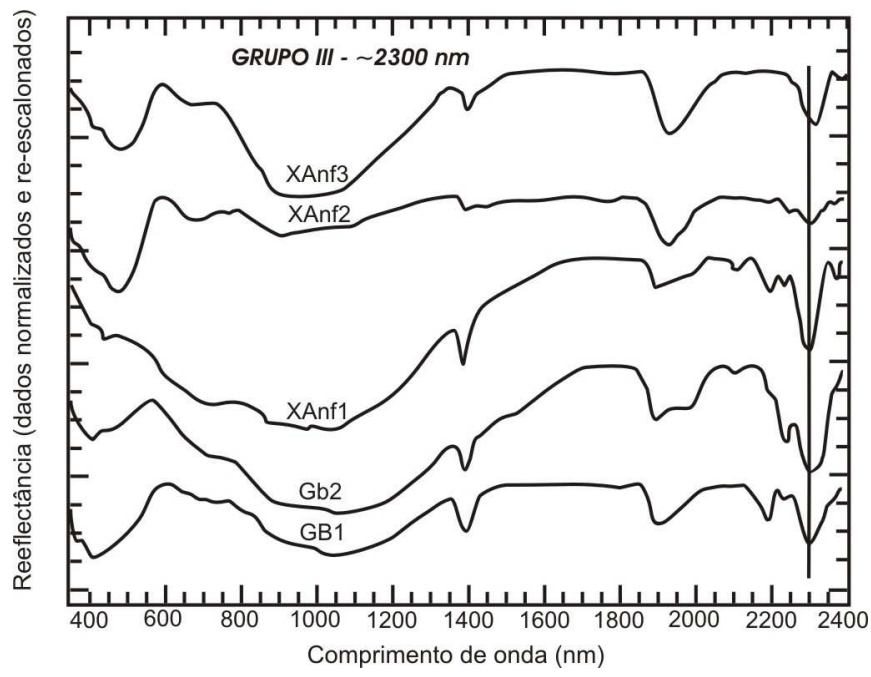

Figura 6 - Grupo Espectral III, com feições de absorção entre 2300nm e 2400nm (GB = amostras de gabro; XAnf = amostras de anfibólio-xistos laminados da Unidade B).

\section{DEPÓSITO SALOBRO UTILIZANDO DADOS GEOSCAN}

Utilizando o modelo exploratório adaptado ao depósito Salobro (Tabela 3) foi realizado o processamento digital das imagens GEOSCAN. Este processamento foi subdividido em dois conjuntos de técnicas: o primeiro, voltado ao realce e discriminação (técnicas "convencionais"/ "tradicionais") entre materiais geológicos; e o segundo, visando a identificação sistemática (classificação espectral) desses materiais.

Discriminação dos materiais geológicos Para o realce da vegetação presente no depósito Salobro foi utilizada uma composição colorida falsa-cor entre as bandas 17/8/2 (RGB) (Fig. 7). A banda 8 , situada na região do infravermelho próximo $(873 \mathrm{~nm}$ $\pm 22 \mathrm{~nm}$ ), detecta um alto de reflectância típico da vegetação (o que também pode ser detectado nas bandas GEOSCAN de 6 à 10). Estando alocada ao canal verde, esta banda permite caracterizar, em tons de verde, áreas onde há presença dominante de matas ciliares (verde mais claro), árvores, arbustos e gramíneas, independente se fotossinteticamente ativas ou não. A banda 2, que cobre o intervalo do verde do espectro visível $(583 \pm 67 \mathrm{~nm})$ e foi aqui alocada ao canal azul, mapeia alterações de reflectância devido à variações de pigmento (e.g., clorofila) na vegetação (Clark, 1999). Essas alterações, que teoricamente são sutis, também pouco aparecem na imagem. A banda 17, posicionada no infra-vermelho de ondas curtas $(2308 \mathrm{~nm} \pm 44 \mathrm{~nm})$, é capaz de discriminar áreas onde rochas e solos estão expostos, além de detectar variações de reflectância na vegetação devido à presença de compostos bioquímicos resistentes (i.e., lignina, celulose e proteína - Clark 1999), podendo indicar áreas com predomínio de vegetação mais seca na imagem. No seu conjunto, a composição colorida 17/8/2 (RGB) (Fig. 7) aponta a presença de vegetação verde e seca recobrindo, quase que por completo, a área do depósito Salobro. Os setores em tons de magenta, ou mesmo vermelho, nessa imagem apresentam exposição total ou parcial de solos e rochas. Tais áreas estão associadas a superfícies ferruginosas formadas por processos de intemperismo das formações ferríferas e do minério (B), por quartzitos do Grupo Macaúbas (A), e áreas com ocupação 
Modelo exploratório para a prospecção de depósitos de Zn-Pb utilizando dados de alta resolução espacial e espectral do sensor Geoscan MKII: estudo de cado no depósito Salobro, Porteirinha (MG)

Tabela 3 - Intervalos espectrais e bandas do sensor GEOSCAN MKII selecionadas para a detecção das principais feições do depósito Salobro por sensoriamento remoto.

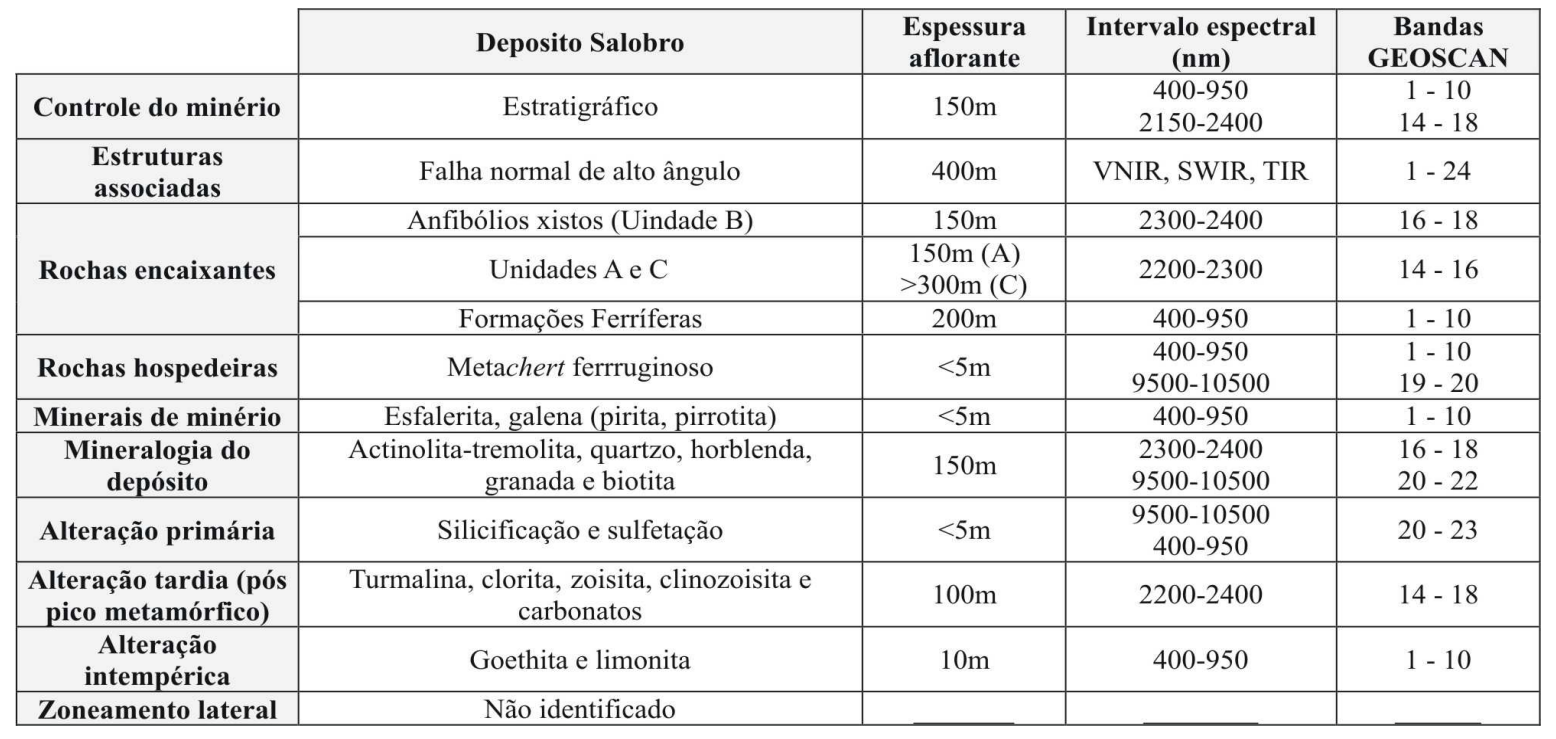

antrópica (C e D).

A composição colorida falsa-cor 20/14/6 (RGB) foi projetada para o realce de materiais ricos em sílica (banda $20-9170 \mathrm{~nm} \pm$ $530 \mathrm{~nm}$ ), minerais portadores da molécula hidroxila (banda 14 $2176 \mathrm{~nm} \pm 44 \mathrm{~nm}$ ) e superfícies ricas em hidróxidos de ferro (íon $\mathrm{Fe}^{3+}$ ) (banda $6740 \mathrm{~nm} \pm 23 \mathrm{~nm}$ ), em tons de vermelho, verde e azul, respectivamente. Adicionalmente, essa composição colorida foi sobreposta ao modelo digital de terreno da área na qual o depósito Salobro encontra-se inscrito (Fig. 8). Essa imagem individualizou o contato entre o Grupo Macaúbas e a Seqüência Salobro, porém não foi uma composição adequada para a separação desta seqüência do embasamento. Na porção centro-oeste da Seqüência Salobro ocorrem áreas contendo pixels que variam entre tons de vermelho e magenta em cristas convexas (A, B e C), indicando que se tratam de superfícies ricas em sílica e hidróxidos de ferro. Estas anomalias apresentam-se alinhadas ao trend da foliação principal e correspondem à expressão superficial das formações ferríferas bandadas e do nível de metachert ferruginoso mineralizado em $\mathrm{Zn}-\mathrm{Pb}$. Esta combinação de bandas, construída com base no modelo exploratório, mostrou-se muito eficaz para a discriminação do nível sulfetado e formações ferriferas magnéticas, tipos litológicos com estreitas relações genéticas.

Identificação dos materiais geológicos A classificação espectral de imagens de sensoriamento remoto baseia-se na comparação entre a assinatura espectral dos pixels de composição desconhecida (imagem) e a assinatura espectral dos materiais de referência (bibliotecas espectrais). Esta biblioteca pode incluir espectros de minerais puros, misturas de alguns minerais, o espectro global de uma rocha ou pixels da própria imagem, para os quais a composição é conhecida. Dois métodos para o mapeamento espectro-mineralógico foram aplicadas nas imagens GEOSCAN visando a identificação mineralógica no depósito Salobro: o método SAM (Spectral Angle Mapper, Kruse et al. 1993) e o método SFF (Spectral Feature Fitting, Boardman \& Kruse 1994). Estes métodos, desenvolvidos inicialmente para dados hiperespectrais, têm sido adaptados com sucesso para a classificação de dados multiespectrais, incluindo dados do sensor GEOSCAN (Fraser \& Agar 1997, Agar \& Pávez 1999).
O SAM é uma técnica de classificação supervisionada que mede a similaridade espectral entre o espectro de reflectância real de cada pixel e o de um espectro de referência. Esta técnica trata os espectros desconhecidos da imagem e os espectros de referência como vetores em um espaço cuja dimensionalidade é igual ao número de bandas espectrais do sensor. No procedimento de classificação, o algoritmo do SAM determina a similaridade entre duas curvas espectrais através do cálculo do ângulo espectral quanto menor for o ângulo entre os vetores, maior similaridade espectral será atribuída entre o pixel analisado e o material de referência em questão. Uma das limitações deste processo é que somente a direção é considerada para os espectros e não o seu comprimento, o que torna o método insensível a fatores de ganho e a todas as possíveis diferenças de iluminação da cena - os pixels escuros cairão sempre próximos a origem do espaço n-dimensional.

Utilizando os espectros de referência das formações ferríferas magnéticas e não-magnéticas e do minério como biblioteca espectral (endmembers), foram gerados resultados através do SAM para o mapeamento dos pixels referentes a cada uma destas rochas. Dois resultados foram derivados do SAM. Um primeiro refere-se a um conjunto de imagens, denominadas de Rule. Neste caso, para cada espectro de referência utilizado, uma imagem Rule foi gerada, de forma que cada pixel possui valor igual ao ângulo espectral. Nas imagens Rule os pixels originalmente mais escuros apresentam um menor valor para o ângulo entre os espectros e, conseqüentemente, uma maior similaridade entre os mesmos. Desta forma, por uma questão visual, foi necessário que os histogramas referentes às estas imagens fossem cuidadosamente invertidos e ajustados - os pixels originalmente escuros foram convertidos em mais claros, ficando destacados na cena. O segundo resultado gerado foi um mapa de classificação final. Esse mapa é baseado na similaridade espectral dos pixels da imagem com os materiais de referência selecionados; em geral os resultados que figuram nesse mapa de classificação são incompletos, o que já não ocorre com a análise das imagens Rule.

As três imagens Rule resultantes do SAM para os três endmembers tiveram seus histogramas invertidos e ajustados e foram combinadas em composição colorida RGB(Fig. 9). A análise do resultado mostra que, na porção centro-oeste da área, a resposta 


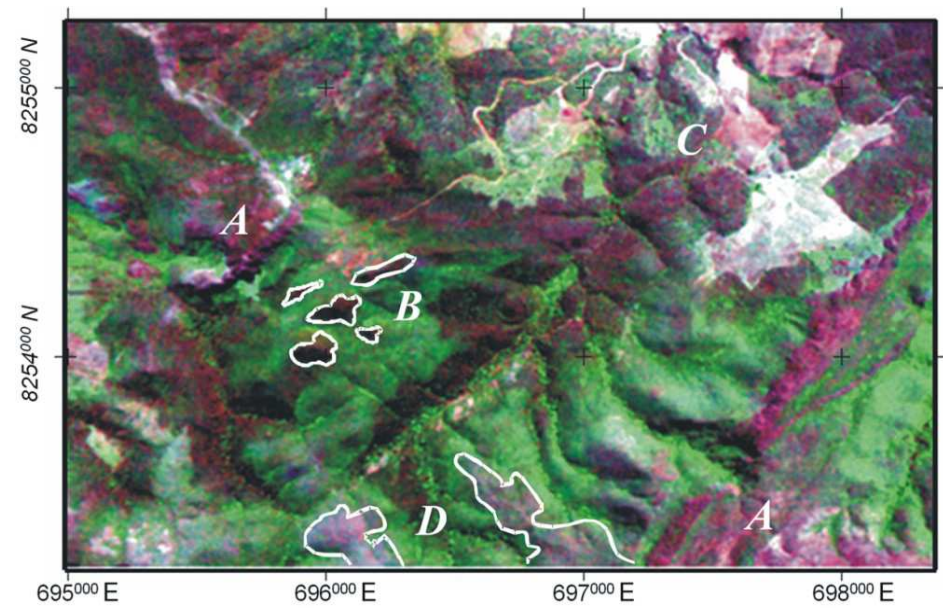

Figura 7 - Composição colorida falsa-cor entre as bandas 17/8/2 ilustrando a distribuição da vegetação na área do depósito Salobro. A: domínio do Grupo Macaúbas; B: domínio das formações ferríferas bandadas e minério; C e D: área com ocupação antrópica.

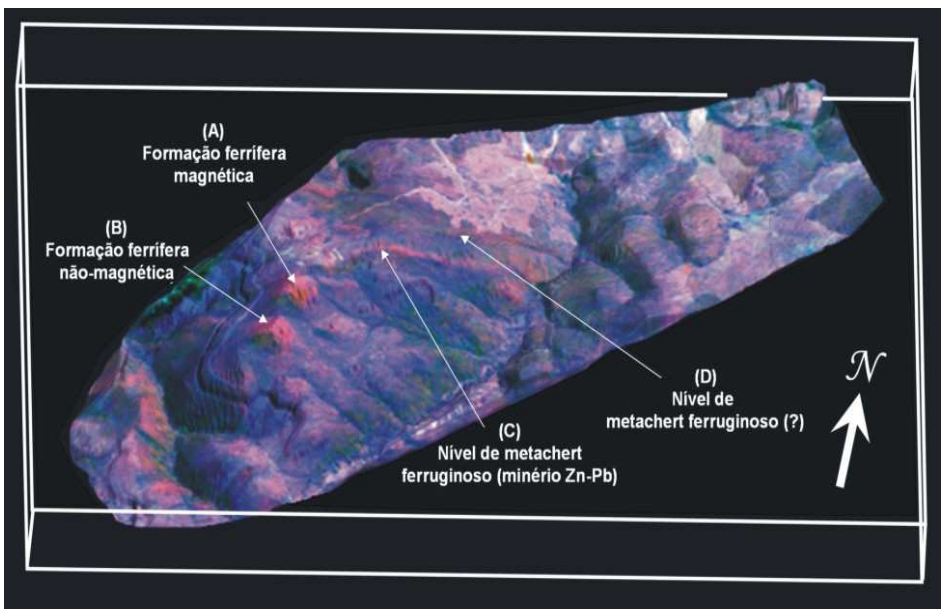

Figura 8 - Imagem falsa-cor entre as bandas 20/14/6, superposta ao Modelo Digital de Terreno da área do depósito Salobro, ilustrando a distribuição de sílica/ minerais portadores da molécula hidroxila / hidróxidos de ferro no depósito Salobro.

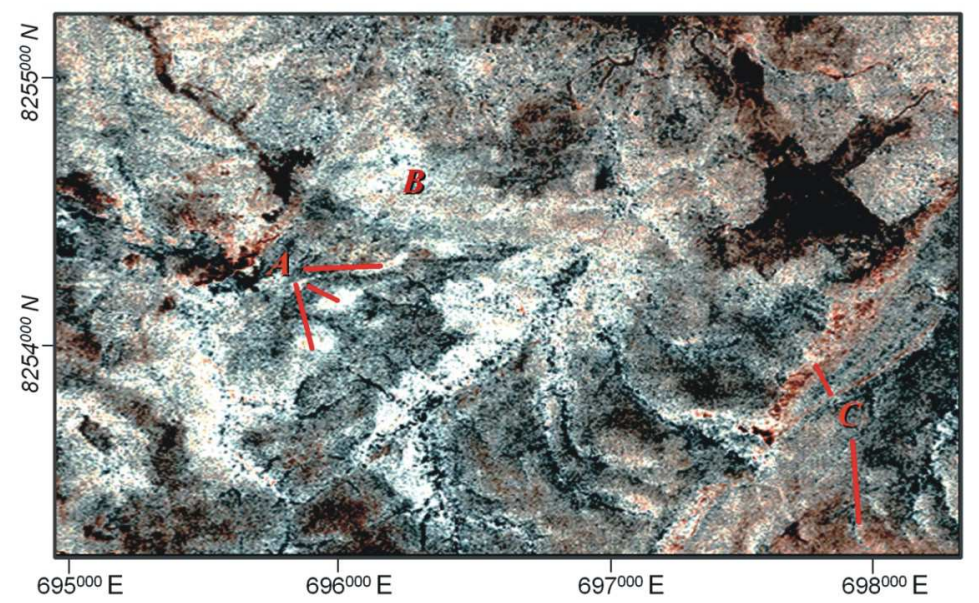

Figura 9 - Classificação espectral pelo método Spectral Angle Mapper. As curvas espectrais das formações ferríferas não-magnética Imagnética/minério foram utilizadas como endmembers no processo de classificação. As imagens Rule para cada um dos endmembers foram alocadas, respectivamente, aos canais $R G B$, resultando na imagem de abundância relativa de cada material. 

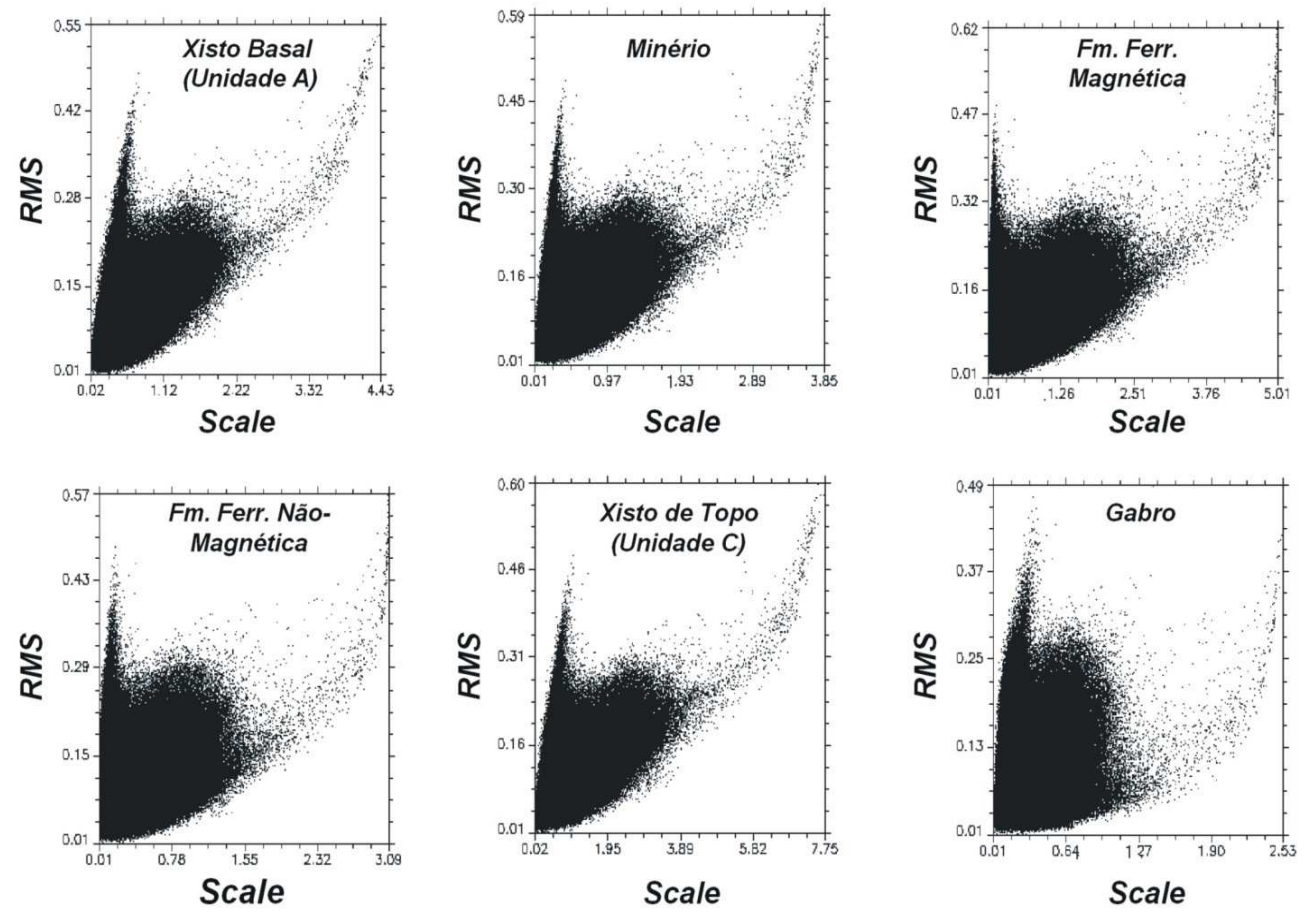

Figura 10 - Gráficos de dispersão entre as imagens RMS e Scale gerados a partir da classificação espectral SFF (Spectral Feature Fitting).

espectral das formações ferríferas e do minério contribuem proporcionalmente nos pixels da imagem (coloração esbranquiçada - A). Entretanto, não é possível delimitar e distinguir os contatos e o predomínio de um litotipo em relação ao outro, por esta técnica. Além disto, pixels esbranquiçados se espalham por toda a imagem, como na porção norte da área (B), onde estas rochas não ocorrem. Pixels em tons vermelho também encontram-se difusamente espalhados, por razões ainda desconhecidas, no domínio das rochas do Grupo Macaúbas e nas zonas de cisalhamento (C). A técnica SAM, de forma geral, não apresentou bons resultados, provavelmente em função da qualidade da transformação dos dados GEOSCAN para reflectância pelo método IARR.

O Spectral Feature Fitting é um método de classificação baseada na identificação das feições espectrais diagnósticas de cada material. A técnica analisa simultaneamente múltiplos materiais, selecionados a partir de espectros de referência utilizando feições diagnósticas de absorção, aumentando assim a capacidade de identificação. Inicialmente, esta técnica remove o contínuo dos pixels da imagem e dos espectros de referência. Cada espectro de referência é então comparado com o espectro desconhecido da imagem, produzindo uma imagem denominada de Scale. A partir daí, os espectros de referência e o da imagem com os contínuos removidos são comparados utilizando um algoritmo por mínimos quadrados e um coeficiente linear de correlação. Desta operação, o erro médio quadrático é determinado para cada espectro de referência, produzindo uma imagem $R M S$. Finalmente, uma imagem (fit) é produzida para cada mineral de referência resultante da divisão da imagem Scale pela do RMS.

Os resultados obtidos no processamento das imagens GEOCAN no depósito Salobro segundo o método SFF não foram satisfatórios. A performance limitada deste método é observada nos scatergramas das imagens scale e $R M S$, onde foram utilizados como endmembers todos os espectros dos litotipos presentes no depósito Salobro (Fig. 10). Nestes diagramas de dispersão é observada uma concentração anômala de pixels ao longo de uma reta que faz um ângulo alto com o eixo RMS. Isto implica que existe um ajuste inadequado entre os pixels da imagem e os espectros de referência, de forma que os resultados da classificação são considerados muito duvidosos e imprecisos.

DISCUSSÕES E CONCLUSÕES As características dos depósitos de $\mathrm{Zn}-\mathrm{Pb}$ do tipo estratiforme hospedado em sedimentos, mais facilmente detectadas por sensoriamento remoto, relacionam-se aos produtos do hidrotermalismo atuante sobre as rochas sedimentares hospedeiras (alterações primárias e secundárias no pipe, zoneamento lateral e mineralogia de alteração) e transformações metamórficas posteriores. Isto ocorre neste tipo de depósito pois as seqüências sedimentares hospedeiras não são únicas, variando desde metacherts até calcários, os quais apresentam respostas variadas nas bandas do sensor sob análise - algumas diagnósticas, outras não.

Assim, a adequação do modelo prospectivo realizando um estudo prévio sobre a área de interesse, aumenta a possibilidade de detecção correta de alvos metalogeneticamente importantes. A interpolação de características geológicas, petrográficas e espectrais das unidades aflorantes na área de interesse, com os aspectos fisiográficos que as controlam, pode ser um fator decisivo para o mapeamento dos alvos de prospecção.

No caso do depósito Salobro, os fatores fisiográficos da região inibiram o alcance de melhores resultados a partir do processamento das imagens GEOSCAN, principalmente no tocante à delimitação da seqüência hospedeira do minério de $\mathrm{Zn}-\mathrm{Pb}$. O recobrimento quase total da Seqüência Salobro por uma densa vegetação (que mesmo seca apresenta uma resposta espectral específica); a 
presença de um espesso nível coluvionar (de aproximadamente $1 \mathrm{~m}$ de espessura); e ação da alteração intempérica, mascaram, quase por completo, a real resposta espectral do substrato rochoso compreendido pela Seqüência Salobro.

A estratégia do processamento dos dados GEOSCAN no depósito Salobro, portanto, foi primeiramente dirigida para o mapeamento de regiões que não apresentam cobertura vegetal e cobertura coluvionar (e.g. altos topográficos). A imagem falsa cor entre as bandas 17, 8 e 2 (RGB) permitiu cartografar a distribuição da vegetação no depósito. Nesta imagem foi possível delimitar pequenas áreas, dentro da seqüência Salobro, que apresentam solo e rochas expostas. No produto gerado pela fusão do modelo digital de terreno à imagem falsa cor que representa simultaneamente as concentrações anômalas de sílica, hidroxilas e óxidos e hidróxidos de ferro (bandas 20, 14 e 6 - RGB), foi observado que tais áreas, isentas de vegetação, no interior da seqüência, apresentam anomalias significativas de sílica e íons de ferro. Essas áreas, quando comparadas com a geologia da área, coincidem plausivelmente com as expressões superficiais do nível de metachert ferruginoso (rocha hospedeira da mineralização) e formações ferríferas bandadas. Esse resultado é extremamente importante pois provavelmente trata-se de um dos poucos casos conhecidos na literatura de sensoriamento remoto geológico onde não só rochas indiretamente associadas a mineralização, mas também, o corpo do minério, foi caracterizado por sensores remotos numa região de clima tropical.

Agradecimentos À CVRD-DOCEGEO, pela disponibilização dos dados e suporte logístico em Janaúba-MG. T. Almeida agradece ao Conselho Nacional de Pesquisa $(\mathrm{CNPq})$ pela bolsa de estudos de Mestrado. C.R. Souza Filho agradece o suporte do CNPq e FAPESP, respectivamente, pela bolsa de Produtividade em Pesquisa (Proc. No. 301.227/94) e pelo Auxílio à Pesquisa Jovem Pesquisador (Proc. No. 96/11139-2).

\section{Referências}

Abreu F.R. \& Oliveira O.A.B. de 1998. Geologia e ocorrências de zinco e chumbo do Deposito Salobro, Porteirinha-MG. In: Congr. Bras. Geol., 40, Belo Horizonte, 1998. Anais,SBG. 1, p.140.

Agar B. \& Pavez A. 1999. Archival data; "Old dogs with new tricks?". In: $13^{\text {th }}$ International Conference on Applied Geologic Remote Sensing, Vancouver, Canadá. Proceddings 1: 1-8.

Almeida T. 2000. Modelo Exploratórios para a prospecção de Zn-Pb utilizando dados de sensoriamento remoto: Estudo de caso do Deposito Salobro (Porteirinha-MG). 132p. (Dissertação de mestrado, Instituto de Geociências, Universidade Estadual de Campinas).

Boardman J.W. \& Kruse F.A. 1994. Automated spectral analysis: A geologic example using AVIRIS data, north Grapevine Mountains, Nevada. In: $10^{\text {th }}$ Thematic Conference on Geologic Remote Sensing, Environment Research Institute of Michigan, Ann Arbor, MI, I407I418.

Chenkui C., Xianfang H., Fusheng Z.D.L., Jun G., Guojuan W., Zhenguo J., Xialio Y., Song F. 1991. Deposit Image Model and optimizationa of prospect tarets. In: $8^{\text {th }}$ Thematic Conference on Geologic Remote Sensing, Denver, Colorado (USA). Proceddings: 605-612.

Clark R.N. 1999. Spectroscopy of rocks and minerals, and Principles of Spectroscopy. http://speclab.cr.usgs.gov/PAPERS.refl-mrs/refl4.html. 14/06/1999.

Conel J.E., Green R.O., Vane G., Bruegge C.J., Alley R.E., Curtiss B.J. 1987. Airbone imaging spectrometer-2: radiometric spectral characteristes and comparision of ways to compensate for the atmosphere. In: SPIE, 834: 140-157.

Crocco-Rodrigues F.A., Vidigal M.L., Carneiro N.M.. Greco S.M.C., Freitas M.E., Costa W.D. 1992. Mapeamento Geológico- Estrutural em escala de 1:50.000 da região de Riacho dos Machados (MG). Relatório interno DOCEGEO - Geologia e Mineração.

Curtiss B. \& Goetz A.F.H. 1994. Field Spectrometry: Techniques and Intrumentation. In: Proceedings of the International Symposium on Spectral Sensing Research, Boulder-Co: 31-40.

Daitx E.C. 1996. Origem e evolução dos depósitos sulfetados tipo Perau (Pb-Zn-Ag) com base nas jazidas Canoas e Perau (Vale do Ribeira, $P R$ ). 431p. (Tese de Doutorado, Instituto de Geociências e Ciências Exatas, Universidade Estadual Paulista, Rio Claro, S.P.).
Dardenne M.A. \& Freitas-Silva F.H. 1999. Pb-Zn ore deposits of Bambuí and Vazante Groups, in São Francisco Craton and Brasilia Fold Belt, Brazil. In M.G. Silva e A. Misi (eds.) Base Metal Deposits of Brazil. Belo Horizonte, MME/CPRM/DNPM, 75-83.

DOCEGEO. 2001. Relatório Anual do Projeto Riacho dos Machados. DOCEGEO (documento interno).

Drumond J.B.V., Sperling E.V., Raposo F.O. 1980. Projeto PorteirinhaMonte Azul (1:50.000). Relatório final. I DNPM/CPRM, Inédito, $559 \mathrm{p}$.

Du M. 1996. Mineral mapping at El Abra cooper Deposit, Chile, using GEOSCAN 24-Channel scanner data and spectral angle mapper classifier. In: $11^{\text {th }}$ Thematic Conference and Workshops on Applied Geologic Remote Sensing, Las Vegas. Proceddings I: 385-391.

Fleischer R. \& Espourteille F.S. 1999. The Boquira lead-zinc mine in Central Bahia, Brazil. In M.G. Silva e A. Misi (eds.) Base Metal Deposits of Brazil. Belo Horizonte, MME/CPRM/DNPM, p. 4453.

Fraser S.J. \& Agar R.A. 1997. GIS Integration of Airbone Multiespectral, Geophysical and other Mineral Exploration Data at the el Halcon Porphyry Cooper Prospect, Copiapo, Chile. In: $12^{\text {th }}$ International Conference and Workshops on Applied Geologic Remote Sensing. Denver, Colorado. Proceedings II: 245-252.

Gooddfellow W.D., Lyndon J.W., Turner R.J.W. 1993. Geology and Genesis of Stratiform Sediment Hosted (SEDEX) Zinc-Lead-Silver Sulphide Deposits. In Kirkham, R.V.; Sinclair, W.D.; Thorpe, R.I. \& Duke, J.M.(eds.), Mineral Deposits Modeling: Geological Association of Canada, Canadá, Special Paper 40: 201-251.

Grove C.I., Hook S., Paylor E.D. 1992. Laboratory reflectance Spectra of 160 minerals 0.4 to 2.5micrometer: JPL Publication 92-2, Pilot Land Data System, Jet Propulsion Laboratory, Pasadena, California.

Guilbert J.M. \& Park C.F. 1986. Deposits related to submarine volcanism and sedimentation. Freeman and Company (ed), The Geology of Ore Deposits. New York, 660-675.

Guimaraes M.L.V., Crocco-Rodrigues F.A., Abreu F. R., Oliveira O.A. B., Greco F.M. 1993. Geologia do Bloco Itacambira-Monte Azul entre Barrocao e Porteirinha (MG). In: SBG/ Núcleo MG, VII Simpósio de Geologia de Minas Gerais, Anais, 12: 74- 78. 
Modelo exploratório para a prospecção de depósitos de Zn-Pb utilizando dados de alta resolução espacial e espectral do sensor Geoscan MKII: estudo de cado no depósito Salobro, Porteirinha (MG)

Gustafson L.B. \& Williams N. 1981. Sediment-hosted stratiform deposits of cooper, lead and zinc. Economic Geology, $75^{\text {th }}$ Anniversary: 139178.

Holm O., Pongratz J., McGoldrick P. 1999. Basins, Fluids and Zn-Pb Ores, CODES Special publication 2.

Kruse F.A. 1990. Artificial Intelligence for analysis of imaging spectrometer data. Proceddings ISPR Commission VII, Working Group 2: "Analysis of Hight spectral resolution imaging data", Victoria, B.C., Canada, 17-21 September 1990. 59-68.

Kruse F.A., LefkoffA.B., Dietz J.B. 1993. Expert System-Based Mineral Mapping in Nothern Death Valley, California/Nevada using the airbone visible/infrared Imaging Spectrometer (AVIRIS). Remote Sensing of Environment, Special issue on AVIRIS, May-June, 309-336.

Largie D.A., Lockett N.H., Agar R.A.A., KroenkeM., Lyon R.J.P. 1993. An integrated LANDSAT- MSS, aeromagnetic, air- photographic and GEOSCAN AMSS approach to gold exploration in Nevada. In: $9^{\text {th }}$ Thematic Conference on Remote Sensing Exploration, Environment and Engineering, Pasadena, California (USA). Proceedings, 2: 315-322.

Largie D.E. 1983. Sediment-hosted massive sulphide lead-zinc deposits: An empirical model. In: Sangster, D.F., (ed)., Sediment- Hosted Stratiform Lead-Zinc Deposits: Mineralogical Association of Canada, Short Course Handbook, 8: 1-29.

MacIntyre D. 1995. Sedimentary Exhalative Zn-Pb-Ag. In Lefebure, D.V. \& Ray, G.E. (eds.), Selected British Columbia Mineral Deposit Profiles, 1: Metallics and Coal, Ed. British Columbia Ministry of Energy of Employment and Investment, Open File 1995-20, 37-39.

Macklin S. 1998. Simis-Field Version 2.8. Spectrometer Independent Mineral Identification Software. User Manual version 2.8.

Maracci G. 1992. Field and laboratory narrow band spectrometers and the techniques employed. In: Toselli, F. \& Bodechtel, J. (eds.). Imaging Spectrscopy: Fundamentals and Prospective Applications. 33-46.

Passos R.V. 1999. Caracterização da geometria de zonas de alteração hidrotermal-Estudo de caso no depósito aurífero de Brumal, Quadrilátero Ferrifero, MG. 180p. (Dissertação de mestrado, Instituto de Geociências, Universidade Estadual de Campinas.).

PROSPEC-Prospecção e aerolevantamentos 1993. Aerolevantamento multiespectral com o sistema GEOSCANMKII. Área de Baú-Serrinha $-M G$. DOCEGEO- Relatório interno. 66p.

Roberts D.A., Yamaguchi Y., Lyon R.J.P. 1985. Calibration of airbone imaging spectrometer data to percente reflectance using field measurements. Proceedings of the $19^{\text {th }}$ Interantional Symposium on Remote Sensing of Enviroment. 21-25.

Santos L.D. \& Paes V.J.C. 1992. Mapeamento Geológico da Serra do Coco e Imediações, Município de Porteirinha/MG. 27p. (Trabalho de Graduação, IGC-UFMG, Belo Horizonte).

Spatz, D.M. 1999. Remote Sensing characteristics of sediment-hosted stratiform copper deposits. In: $13^{\text {th }}$ International Conference on Applied Geologic Remote Sensing, Vancouver, Canadá. Proceedings, I:214-221.

Spatz D.M. 1997. Remote sensing characteristics of the sediment- and volcanic- hosted precious metal systems: imagery selection for exploration and development. International Journal of Remote Sensing, 18, $\mathrm{n}^{\circ}$ 7: 1413-1438.

Spatz D.M. \& Wilson R.T. 1997. Remote sensing characteristics of the volcanic- associated massive sulfide systems. In: $12^{\text {th }}$ International Conference and Workshops on Applied Geologic Remote Sensing. Denver, Colorado. Proceedings I: 1-12.

Spatz D.M. 1996. Remote sensing characteristics os precious metal systems: The sediment-hosted and detachment related deposits, $11^{\text {th }}$ Thematic Conference on Geologic Remote Sensing, Ann Arbor, Proceedings, I: 13-22.

Swalf P. S. 2000. Modelo Exploratório para depósitos auríferos do tipo Morro do Ouro com base em dados e técnicas de sensoriamento remoto. $118 \mathrm{p}$. (Dissertação de mestrado, Instituto de Geociências, Universidade Estadual de Campinas).

Wilton D. 1998. SEDEX- Type Massive Sulphide Deposits In: http:// www.bc-mining-house.com/prospecting_school/dp_msedx.html.

Manuscrito SR-01

Recebido em 12 de agosto de 2002

Revisão dos autores em 20 de fevereiro de 2003 Revisão aceita em 03 de abril de 2003 